\title{
Borboletas (Lepidoptera: Papilionoidea e Hesperioidea) de um parque urbano em Belo Horizonte, Minas Gerais, Brasil
}

\author{
Glória Ramos Soares ${ }^{1,2,5}$, Andréa Aparecida Paiva de Oliveira ${ }^{3}$ \& André Roberto Melo Silva \\ ${ }^{1}$ Centro Nacional de Pesquisa e Conservação da Biodiversidade do Cerrado e Caatinga - CECAT, \\ Instituto Chico Mendes de Conservação da Biodiversidade - ICMBio, Brasília, DF, Brasil \\ ${ }^{2}$ Pesquisa e Conservação do Cerrado - Pequi, SCLN 408, Bloco E, Sala 201, \\ CEP 70856-550, Brasília, DF, Brasil \\ ${ }^{3}$ Departamento de Manejo, Fundação de Parques Municipais de Belo Horizonte, \\ Belo Horizonte, MG, Brasil \\ ${ }^{4}$ Faculdade de Ciências Biológicas e da Saúde, Centro Universitário UNA, Rua Guajajaras, 175, \\ Centro, CEP 30180-100, Belo Horizonte, MG, Brasil \\ ${ }_{5}^{5}$ Autor para correspondência: Glória Ramos Soares, e-mail: biogloria@yahoo.com.br
}

SOARES, G.R., OLIVEIRA, A.A.P. \& SILVA, A.R.M. Butterflies (Lepidoptera: Papilionoidea and Hesperioidea) from an urban park in Belo Horizonte, Minas Gerais State, Brazil. Biota Neotrop. 12(4): http://www.biotaneotropica.org.br/v12n4/en/abstract?inventory+bn02612042012

\begin{abstract}
Urban forest fragments, such as parks and squares provide food resources, shelter, and favorable conditions for the maintenance of butterflies. This study provides an inventory of butterflies and observations of plants visited by adults in a small urban park, the Américo Renê Giannetti Municipal city park, Belo Horizonte, Minas Gerais State. We sampled an area of 18.2 ha from December 2006 to December 2007 with entomological net and traps baited with fermented fruits. We recorded 165 individuals belonging to 78 species, as follows: 46 spp. of Nymphalidae; 10 spp. of Hesperiidae, seven spp. of Lycaenidae, seven spp. of Pieridae, five spp. of Riodinidae; and three species of Papilionidae. Between the plants visited the more attractive was Lantana camara (Verbenaceae), a shrub with flowers of vibrant colors and common source of nectar and pollen. The butterflies recorded show the importance of maintenance of urban green areas, providing favorable conditions for the permanence of butterflies, even in anthropic environments.
\end{abstract}

Keywords: inventory, insect-plant interactions, butterfly conservation, urban forest, Lantana camara.

SOARES, G.R., OLIVEIRA, A.A.P. \& SILVA, A.R.M. Borboletas (Lepidoptera: Papilionoidea e Hesperioidea) de um parque urbano em Belo Horizonte, Minas Gerais, Brasil. Biota Neotrop. 12(4): http://www. biotaneotropica.org.br/v12n4/pt/abstract?inventory+bn02612042012

Resumo: Fragmentos urbanos de mata, como parques e praças, oferecem recursos alimentares, sobretudo abrigo e condições favoráveis para a permanência de borboletas. Este estudo fornece um inventário de borboletas e também a observação das plantas visitadas por indivíduos adultos em um pequeno parque urbano, Parque Municipal Américo Renê Giannetti, Belo Horizonte, estado de Minas Gerais. Amostrou-se uma área de 18,2 ha no período de dezembro/2006 à dezembro/2007, com o auxilio de rede entomológica e armadilhas com frutos fermentados. Foram registrados 165 indivíduos de 78 espécies, distribuídos nas seguintes famílias: Nymphalidae - 46 spp., Hesperiidae - 10 spp., Lycaenidae - sete spp., Pieridae - sete spp., Riodinidae - cinco spp. e Papilionidae - três spp. Entre as plantas visitadas observou-se que a com maior atratividade foi Lantana camara (Verbenaceae), um arbusto com flores de cores vibrantes, destacada como fonte usual de néctar e pólen. As borboletas encontradas indicaram a importância da manutenção de áreas verdes urbanas, proporcionando condições favoráveis para a permanência de borboletas, mesmo em ambientes antrópicos.

Palavras-chave: inventário, interação inseto-planta, conservação de borboletas, mata urbana, Lantana camara. 


\section{Introdução}

Os insetos compreendem cerca de $59 \%$ de todos os animais do planeta (751.000 espécies) e desempenham papel chave nos ecossistemas terrestres (Wilson 2003). Além da participação em processos como decomposição, ciclagem de nutrientes, produtividade secundária, fluxo de energia e polinização, eles estão envolvidos na dispersão e predação de sementes (Freitas et al. 2003). Atuam também na regulação de populações de plantas e de outros animais, bem como em diversas interações ecológicas (Leppik 1957, Stebbins 1970, Price 1984, Didham et al. 1996).

O uso de alguns grupos de artrópodes como indicadores biológicos, especialmente os insetos, tem sido útil devido a sua grande diversidade e abundância, facilidade de amostragem e rápidas respostas a alterações ambientais. Segundo Lewinsohn et al. (2005), várias discussões tem ocorrido sobre a utilização de bioindicadores na avaliação e monitoramento de habitats. Os autores ressaltam que diferentes grupos funcionais e/ou taxonômicos fornecem informações relevantes para a efetiva conservação e o uso sustentável dos recursos naturais. Afirmam ainda, que de maneira geral, os invertebrados apresentam respostas demográficas e dispersivas mais rápidas do que organismos com ciclo de vida mais longo. Todas estas características sinalizam que estudos mais refinados podem ser realizados com estes insetos, proporcionando a tomada de decisões mais acertadas em planos de conservação.

Constituída por borboletas e mariposas, a ordem Lepidoptera possui cerca de 146 mil espécies descritas com estimativa de 255.000 espécies a serem descobertas (Heppner 1991). Na região Neotropical, as borboletas são representadas entre 7.100 (Beccaloni \& Gaston 1995) e 7.900 espécies (Heppner 1991, Lamas 2004), cujos adultos normalmente são coloridos e de hábito diurno. O Brasil, devido a grande diversidade desses insetos, representa em torno da metade da riqueza neotropical, com 3.300 espécies (Beccaloni \& Gaston 1995, Brown \& Freitas 1999). Além disso, são utilizadas em monitoramentos por responderem rapidamente a modificações ambientais, serem diversas, relativamente fáceis de amostrar e identificar, e possuírem algumas espécies comuns o ano inteiro (Freitas et al. 2003). Por possuírem reprodução rápida, estreitas associações com habitats e plantas hospedeiras, bem como fatores físicos específicos do habitat, são sensíveis a impactos ambientais de diferentes escalas (New et al. 1995). No Brasil, são objetos de muitos estudos científicos (Brown 1996), podendo ainda ser usadas como indicadoras em inventários de fauna e determinação de prioridades, planejamento e administração de reservas naturais (Brown 1992, Brown \& Freitas 2002, Emery et al. 2006, Freitas \& Marini-Filho 2011).

Considerando seu modo de alimentação na fase adulta, as borboletas dividem-se em duas guildas: as frugívoras, que se alimentam principalmente de caldo de frutas fermentadas, e as nectarívoras, que se alimentam de néctar de flores (DeVries 1987). As frutas fermentadas atraem as borboletas através de seu forte odor exalado. Já as flores, atraem os lepidópteros adultos através de recursos como cores, odores, pólen e néctar de alto valor nutritivo (Otero 1986, Brown 1992). Além disso, as borboletas também podem sugar seiva fermentada, sais minerais de poças de água, fezes, urina e carcaças de animais em decomposição (Francini 2010). Há ainda aqueles indivíduos que possuem o aparelho bucal atrofiado, como por exemplo Brassolis sophorae (Linnaeus, 1758) (Nymphalidae), não se alimentando na fase adulta (Ruszczyk \& Carvalho 1993).

A permanência da fauna de borboletas nos habitats, além de ser influenciada pela presença de recursos alimentares, também é explicada por variáveis como sazonalidade, temperatura, perturbação e conectividade entre os fragmentos (Brown \& Freitas 2000b). Koh \& Sodhi (2004) concluíram que o número de espécies de plantas hospedeiras, somado ao isolamento florestal são alguns dos fatores mais relevantes que afetam a riqueza de borboletas em parques urbanos. Uma estratégia interessante que pode ser aplicada em áreas verdes urbanas é o enriquecimento e recomposição vegetal, garantindo assim, recursos e condições favoráveis para a sobrevivência destes insetos.

Agregado à preservação de muitos habitats remanescentes naturais, a criação e manutenção de parques urbanos promove a permanência de espécies nativas, demonstrando ser uma estratégia viável de conservação nas paisagens mais urbanizadas (Koh \& Sodhi 2004). Áreas verdes urbanas promovem a melhora do clima da cidade tornando a temperatura do ambiente agradável, onde grandes arbustos e árvores reduzem a velocidade do vento e a evaporação da umidade do solo (Murphy 1997). Além disto, os parques urbanos possuem o papel de refúgio para as plantas e animais, auxiliando na conservação do ambiente, semelhante à ilhas de habitats margeados por uma matriz inóspita (Primack \& Rodrigues 2001).

Este trabalho apresenta um inventário das borboletas registradas no Parque Municipal Américo Renê Giannetti, em Belo Horizonte, somado as observações eventuais de algumas plantas visitadas pelos indivíduos adultos de borboletas, visando contribuir para o conhecimento da fauna de borboletas da capital mineira.

\section{Material e Métodos}

O Parque Municipal Américo Renê Giannetti (PMARG), situase na região central de Belo Horizonte, Minas Gerais (19 55' 22" S e $\left.43^{\circ} 56^{\prime} 01^{\prime \prime} \mathrm{W}\right)$, ao lado de sua avenida mais movimentada, a avenida Afonso Pena, (Figura 1). Possui uma área de 18,2 ha, composta por flora diversificada de espécies nativas e exóticas, além de vegetação herbácea, capoeiras, árvores frutíferas, bambuzais, plantas ornamentais e um jardim com flores atrativas para borboletas. A capital é caracterizada por um clima tropical de altitude (Cwa, segundo Köppen) com inverno frio e seco e verão quente e úmido. A temperatura média anual é de $21{ }^{\circ} \mathrm{C}$ e a precipitação anual é de aproximadamente $1500 \mathrm{~mm}$ (Instituto... 2011).

Para a amostragem foram utilizadas as técnicas de rede entomológica e armadilhas atrativas com frutos fermentados. Diversos estudos recomendam que as coletas com rede devam abranger os períodos da manhã e da tarde (Pollard 1977, Iserhard \& Romanowski 2004, Marchiori \& Romanowski 2006, Dessuy \& Morais 2007, Paz et al. 2008), todavia, optou-se por coletar apenas no período da tarde por questões logísticas. As amostragens com rede foram realizadas semanalmente, entre dezembro/2006 e dezembro/2007, sempre das 13 h00 às 15 h00, acumulando 104 horas de esforço amostral. Para a coleta das borboletas frugívoras, uma unidade amostral composta por cinco armadilhas (modelo Van Someren-Rydon) foi instalada entre maio e dezembro de 2007. As armadilhas foram posicionadas $1,5 \mathrm{~m}$ acima do solo e a $25 \mathrm{~m}$ de distância entre si, iscadas com banana amassada, misturada a caldo de cana, fermentada por 96 horas. Estas permaneceram abertas durante sete dias mensais, acumulando um esforço amostral de 6.720 horas.

Após a captura, as borboletas foram coletadas e acondicionadas em envelopes entomológicos, sendo posteriormente montadas e depositadas na coleção de invertebrados do Parque Municipal Américo Renê Giannetti, onde foram identificadas. A identificação das borboletas foi realizada com auxílio de bibliografia especializada (D’Abrera 1981, 1994, 1995, Brown 1992, Canals 2003, Willmott 2003, Tyler et al. 1994), além da consulta a especialistas e a coleção do acervo do Centro Universitário UNA. A nomenclatura taxonômica das borboletas foi atualizada de acordo com Lamas (2004) e Wahlberg et al. (2009). 


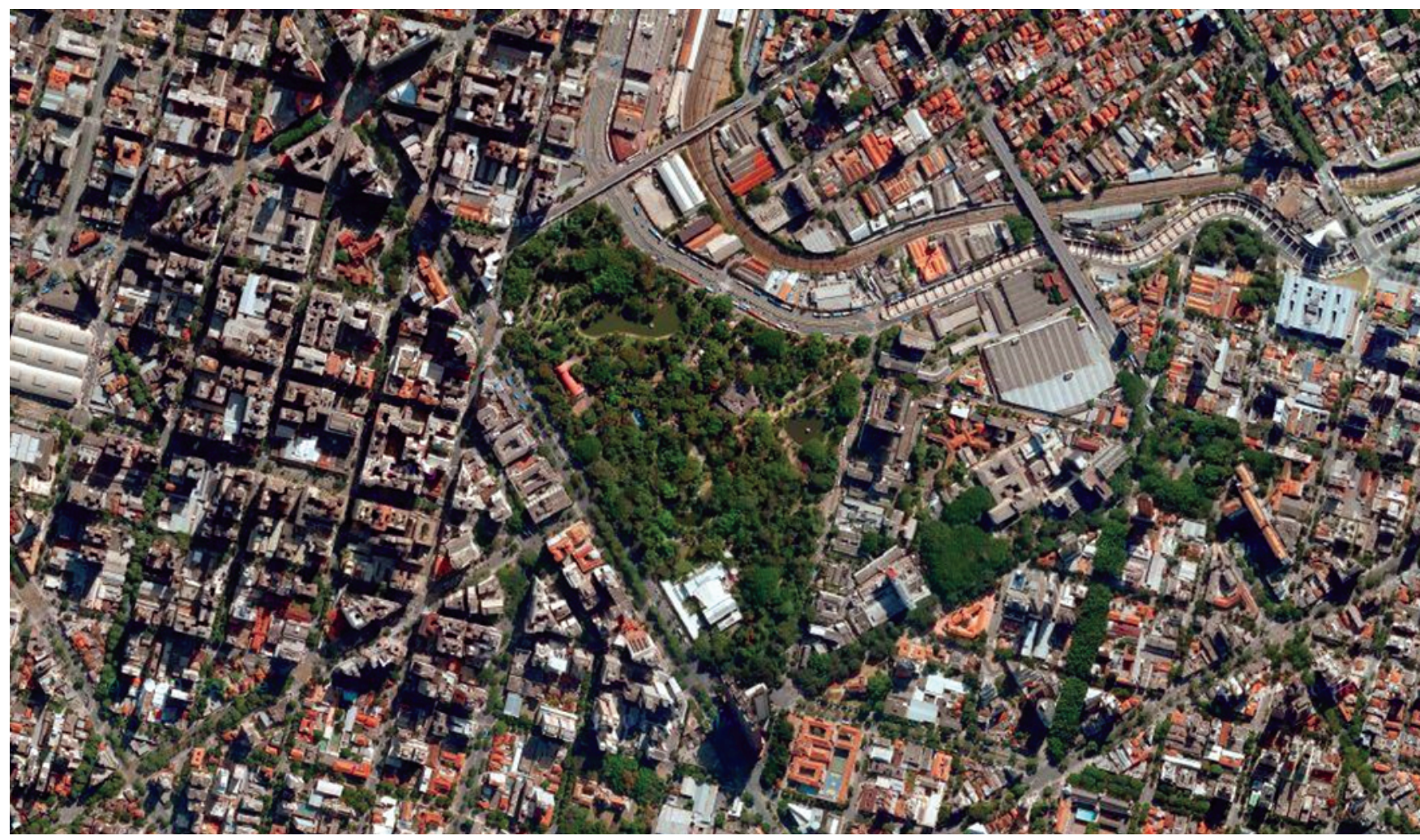

Figura 1. Imagem de satélite da região metropolitana de Belo Horizonte indicando a localização do Parque Municipal Américo Renê Giannetti.

Figure 1. Satellite image in the metropolitan area of Belo Horizonte indicating the location of Américo Renê Giannetti Municipal city park.

Observou-se ainda, de maneira eventual, a visitação floral por borboletas em espécies vegetais presentes no Parque. Estas observações foram realizadas simultaneamente a amostragem de borboletas onde registrou-se as espécies deste grupo que utilizavam as flores como recurso alimentar. Assim, as plantas consideradas mais atrativas foram aquelas que apresentaram o maior número de registros de diferentes espécies de borboletas utilizando suas flores como alimento. A identificação dos espécimes das plantas foi realizada mediante consulta a Lorenzi \& Souza (1995), Souza \& Lorenzi (2005) e a coleção de plantas do PMARG. Daquelas não identificadas no campo, um ramo floral foi coletado para consulta posterior ao biólogo especialista na flora do parque e estão depositadas nesta coleção. A nomenclatura dos vegetais esta de acordo com Angiosperm Phylogeny Website (Stevens 2001).

Para a análise dos dados foi plotado a curva de acumulação de espécies, levando em consideração a riqueza acumulada por mês de coleta.

\section{Resultados e Discussão}

Foram coletados 165 indivíduos de 78 espécies, pertencentes a seis famílias e 16 subfamílias de borboletas (Tabela 1).

Vários esforços têm sido aplicados atualmente em inventários de borboletas no Brasil, contemplando as mais diversas regiões, como a Mata Atlântica, o Cerrado, o Pantanal e os Campos Sulinos (Santos et al. 2008). Este último, de acordo com Santos et al. (2008) é o bioma mais conhecido, possuindo estudos completos da fauna de borboletas através de informações de registros históricos de coletas provenientes da primeira metade do século XX. Porém, em estudo recente no Pampa da região do extremo oeste do Rio Grande do Sul, Rosa et al. (2011) encontraram um registro novo para o Brasil (o pierídeo Tatochila mercedis vanvolxemii (Capronnier, 1874)), evidenciando a necessidade de maiores esforços de amostragem e inventários localizados neste bioma. A Caatinga é totalmente desconhecida nestes inventários, tornando-se o bioma brasileiro mais carente de informação (Santos et al. 2008). Ainda de acordo com Santos et al. (2008), um dos principais motivos da carência de pesquisa deve-se à escassez de estudos de conhecimento básico como diversidade, taxonomia, bionomia e estudo relacionados a níveis populacionais.

Brown \& Freitas (2000b) compilaram registros que resultaram em mais de 2.100 espécies de borboletas para a Mata Atlântica. Segundo estes mesmos autores, no Brasil, as famílias Lycaenidae, Hesperiidae e Nymphalidae são, respectivamente, as três mais ricas em espécies (Brown \& Freitas 1999). Porém, em alguns inventários realizados no Rio Grande do Sul (Iserhard \& Romanowski 2004, Marchiori \& Romanowski 2006, Sackis \& Morais 2008, Iserhard et al. 2010, Rosa et al. 2011) a ordem se inverte, sendo Nymphalidae a mais rica, seguida de Hesperiidae e Lycaenidae. No presente trabalho, verificaram-se padrões semelhantes a estes estudos, onde a família com maior riqueza foi Nymphalidae (59\%), seguida por Hesperiidae (13\%), Lycaenidae (9\%), Pieridae (9\%), Papilionidae (6\%) e Riodinidae (4\%).

A expressiva riqueza encontrada para Nymphalidae pode ser atribuída ao fato desta família possuir o maior número de espécies, maior diversidade de formas de larvas e utilização de uma ampla gama de plantas hospedeiras (DeVries 1987), além de possuírem uma grande diversidade de hábitos, facilitando sua amostragem. Em ambientes neotropicais, a família Nymphalidae abrange cerca de $25 \%$ a $29 \%$ do total da comunidade de borboletas de uma área (Brown \& Freitas 1999, Freitas et al. 2003, Brown \& Freitas 2000b). 
Soares, G.R. et al.

Tabela 1. Lista de espécies de borboletas observadas no Parque Municipal Américo Renê Giannetti, de dezembro de 2006 a dezembro de 2007 , capturadas com rede entomológica e armadilha de frutas.

Table 1. Species list of butterfly recorded in the Américo Renê Giannetti Municipal City Park between December 2006 to December 2007 captured with entomological net and trap baited.

\begin{tabular}{|c|c|c|c|c|}
\hline \multirow{2}{*}{ Família } & \multirow{2}{*}{ Subfamília } & \multirow{2}{*}{ Espécie } & \multicolumn{2}{|c|}{ Método de coleta } \\
\hline & & & Rede & Armadilha \\
\hline \multirow[t]{10}{*}{ Hesperiidae } & Hesperiinae & Cantha ivea Evans, 1955 & $\mathrm{X}$ & \\
\hline & Pyrginae & Achlyodes busirus rioja Evans, 1953 & $\mathrm{X}$ & \\
\hline & & Anastrus sempiternus simplicior (Möschler, 1877) & $\mathrm{X}$ & \\
\hline & & Astraptes anaphus anaphus (Cramer, 1777) & $\mathrm{X}$ & \\
\hline & & Heliopetes alana (Reakirt, 1868) & $\mathrm{X}$ & \\
\hline & & Pyrgus orcus (Stoll, 1780) & $\mathrm{X}$ & \\
\hline & & Urbanus dorantes (Stoll, 1790) & $\mathrm{X}$ & \\
\hline & & Urbanus proteus proteus (Linnaeus, 1758) & $\mathrm{X}$ & \\
\hline & & Urbanus teleus (Hübner, 1821) & $\mathrm{X}$ & \\
\hline & & Xenophanes tryxus (Stoll, 1780) & $\mathrm{X}$ & \\
\hline \multirow[t]{7}{*}{ Lycaenidae } & Polyommatinae & Hemiargus hanno (Stoll, 1790) & $\mathrm{X}$ & \\
\hline & & Leptotes cassius (Cramer, 1775) & $\mathrm{X}$ & \\
\hline & Theclinae & Panthiades hebraeus (Hewitson, 1867) & $\mathrm{X}$ & \\
\hline & & Pseudolycaena marsyas (Linnaeus, 1758) & $\mathrm{X}$ & \\
\hline & & Rekoa palegon (Cramer, 1780) & $\mathrm{X}$ & \\
\hline & & Rekoa stagira (Hewitson, 1867) & $\mathrm{X}$ & \\
\hline & & Theritas hemon (Cramer, 1775) & $\mathrm{X}$ & \\
\hline \multirow[t]{21}{*}{ Nymphalidae } & Biblidinae & Callicore pygas thamyras (Ménétriés, 1857) & $\mathrm{X}$ & $\mathrm{X}$ \\
\hline & & Ectima thecla (Fabricius, 1796) & $\mathrm{X}$ & $\mathrm{X}$ \\
\hline & & Hamadryas amphinome amphinome (Linnaeus, 1767) & & $\mathrm{X}$ \\
\hline & & Hamadryas epinome (C. Felder \& R. Felder, 1867) & & $\mathrm{X}$ \\
\hline & & Hamadryas februa februa (Hübner, [1823]) & & $\mathrm{X}$ \\
\hline & & Hamadryas feronia feronia (Linnaeus, 1758) & & $\mathrm{X}$ \\
\hline & & Hamadryas iphthime (Bates, 1864) & & $\mathrm{X}$ \\
\hline & & Hamadryas laodamia (Cramer, 1777) & & $\mathrm{X}$ \\
\hline & Cyrestinae & Marpesia chiron (Fabricius, 1775) & $\mathrm{X}$ & \\
\hline & & Marpesia petreus (Cramer, 1776) & $\mathrm{X}$ & \\
\hline & Danainae & Aeria olena Weymer, 1875 & $\mathrm{X}$ & \\
\hline & & Brevioleria plisthenes (d'Almeida, 1958) & $\mathrm{X}$ & \\
\hline & & Danaus erippus (Cramer, 1775) & $\mathrm{X}$ & \\
\hline & & Dircenna dero celtina Burmeister, 1878 & $\mathrm{X}$ & \\
\hline & & Ithomia drymo Hübner, 1816 & $\mathrm{X}$ & \\
\hline & & Ithomia agnosia zikani D’Almeida, 1940 & $\mathrm{X}$ & \\
\hline & & Mechanitis lysimnia lysimnia (Fabricius, 1793) & $\mathrm{X}$ & \\
\hline & & Mechanitis polymnia casabranca Haensch, 1905 & $\mathrm{X}$ & \\
\hline & & Methona themisto (Hübner, 1818) & $\mathrm{X}$ & \\
\hline & & Oleria aquata (Weymer, 1875) & $\mathrm{X}$ & \\
\hline & & Pteronymia sylvo (Geyer, 1832) & $\mathrm{X}$ & \\
\hline
\end{tabular}


Borboletas de um parque urbano em Belo Horizonte, $\mathrm{MG}$

Tabela 1. Continuação...

\begin{tabular}{|c|c|c|c|c|}
\hline \multirow{2}{*}{ Família } & \multirow{2}{*}{ Subfamília } & \multirow{2}{*}{ Espécie } & \multicolumn{2}{|c|}{ Método de coleta } \\
\hline & & & Rede & Armadilha \\
\hline \multirow[t]{25}{*}{ Nymphalidae } & Heliconiinae & Actinote thalia pyrrha (Fabricius, 1775) & $\mathrm{X}$ & \\
\hline & & Actinote discrepans d'Almeida, 1958 & $\mathrm{X}$ & \\
\hline & & Agraulis vanillae maculosa (Stichel, [1908]) & $\mathrm{X}$ & \\
\hline & & Dione juno juno (Cramer, 1779) & $\mathrm{X}$ & \\
\hline & & Dryas iulia alcionea (Cramer, 1779) & $\mathrm{X}$ & \\
\hline & & Eueides isabella (Stoll, 1781) & $\mathrm{X}$ & \\
\hline & & Heliconius erato phyllis (Fabricius, 1775) & $\mathrm{X}$ & \\
\hline & & Heliconius ethilla narcaea Godart, 1819 & $\mathrm{X}$ & \\
\hline & Limenitidinae & Adelpha erotia (Hewitson, 1847) & $\mathrm{X}$ & \\
\hline & & Adelpha mythra (Godart, [1824]) & $\mathrm{X}$ & \\
\hline & Nymphalinae & Anartia amathea roeselia (Eschscholtz, 1821) & $\mathrm{X}$ & \\
\hline & & Anartia jatrophae (Linnaeus, 1763) & $\mathrm{X}$ & \\
\hline & & Colobura dirce (Linnaeus, 1758) & $\mathrm{X}$ & $\mathrm{X}$ \\
\hline & & Eresia lansdorfi (Godart, 1819) & $\mathrm{X}$ & \\
\hline & & Hypanartia bella (Fabricius, 1793) & $\mathrm{X}$ & \\
\hline & & Junonia evarete (Cramer, 1779) & $\mathrm{X}$ & \\
\hline & & Ortilia ithra (Kirby, 1900) & $\mathrm{X}$ & \\
\hline & & Siproeta stelenes (Linnaeus, 1758) & $\mathrm{X}$ & \\
\hline & & Tegosa claudina (Eschscholtz, 1821) & $\mathrm{X}$ & \\
\hline & Satyrinae & Brassolis sophorae laurentii Stichel, 1925 & $\mathrm{X}$ & \\
\hline & & Eteona tisiphone (Boisduval, 1836) & & $\mathrm{X}$ \\
\hline & & Paryphthimoides phronius (Godart, [1824]) & $\mathrm{X}$ & $\mathrm{X}$ \\
\hline & & Paryphthimoides poltys (Prittwitz, 1865) & $\mathrm{X}$ & $\mathrm{X}$ \\
\hline & & Yphthimoides affinis (Butler, 1867) & $\mathrm{X}$ & $\mathrm{X}$ \\
\hline & & Yphthimoides renata (Stoll, 1780) & $\mathrm{X}$ & $\mathrm{X}$ \\
\hline \multirow[t]{3}{*}{ Papilionidae } & Papilioninae & Battus polydamas polydamas (Linnaeus, 1758) & $\mathrm{X}$ & \\
\hline & & Heraclides anchisiades capys (Hübner,[1809]) & $\mathrm{X}$ & \\
\hline & & Parides anchises nephalion (Godart, 1819) & $\mathrm{X}$ & \\
\hline \multirow[t]{7}{*}{ Pieridae } & Coliadinae & Eurema albula albula (Cramer, 1775) & $\mathrm{X}$ & \\
\hline & & Eurema elathea flavescens (Chavannes, 1850) & $\mathrm{X}$ & \\
\hline & & Phoebis neocypris (Hübner, [1823]) & $\mathrm{X}$ & \\
\hline & & Phoebis philea philea (Linnaeus, 1763) & $\mathrm{X}$ & \\
\hline & & Phoebis sennae marcellina (Linnaeus, 1758) & $\mathrm{X}$ & \\
\hline & & Pyrisitia leuce leuce (Boisduval, 1836) & $\mathrm{X}$ & \\
\hline & Pierinae & Ascia monuste orseis (Godart, 1819) & $\mathrm{X}$ & \\
\hline \multirow[t]{5}{*}{ Riodinidae } & Euselasiinae & Euselasia hygenius occulta (Stichel, 1919) & $\mathrm{X}$ & \\
\hline & & Euselasia euploea (Hewitson, [1855]) & $\mathrm{X}$ & \\
\hline & Riodininae & Eurybia pergaea (Geyer, 1832) & $\mathrm{X}$ & \\
\hline & & Lasaia agesilas (Latreille, [1809]) & $\mathrm{X}$ & \\
\hline & & Synargis sp Hübner, [1819] & $\mathrm{X}$ & \\
\hline
\end{tabular}


Por outro lado, a família Riodinidae caracterizou-se por uma baixa riqueza o que pode estar relacionado à sua suscetibilidade ao ambiente urbano e à associações mutualísticas específicas com formigas (mirmecofilia), além de serem de difícil visualização em campo. Esta última observação pode estar relacionada ao esforço amostral do presente estudo, o qual evidencia uma curva de suficiência amostral (Figura 2) ainda muito ascendente, onde o aumento do esforço poderia acrescentar novos registros da família. Além disto, os riodinídeos, em geral, possuem uma grande proporção de espécies raras em contrapartida à um pequeno número de espécies abundantes (Callaghan 1978).

A utilização de armadilhas na amostragem de borboletas frugívoras resultou na captura de $17 \%$ do total, representando 14 espécies (Tabela 1). A disponibilidade de recursos alimentares possivelmente limitou a guilda de borboletas frugívoras no PMARG, onde estão ausentes muitas espécies típicas desta comunidade como espécies de Prepona, Archaeoprepona, Memphis, Opsiphanes e Morpho helenor, dentre outras. Frutos grandes e carnosos são escassos no interior do Parque, o que provavelmente restringiu a riqueza de espécies dependentes deste recurso. No local, apenas a jaqueira, Artocarpus heterophyllus Lam. (Moraceae) possui estas características. Seus frutos são grandes e carnosos, e ao caírem no solo, permanecem na área e fermentam atraindo pássaros, micos e muitos insetos.

Segundo Iserhard \& Romanowski (2004), inventários com um longo período amostral possibilitam o aumento do número total de espécies registradas e ampliam a detecção de espécies com tamanhos populacionais baixos (espécies raras) sendo também influenciados pela estrutura da comunidade através da mudança de habitat. De acordo com Freitas et al. (2003), no período de sete dias, um esforço relevante deve contemplar de 12 a 14 horas/pessoa. Brown \& Freitas (2000b) afirmam que com esse número de horas, até 50\% das espécies presentes no local de estudo podem ser registradas. No presente estudo, observou-se que a curva de acumulação de espécies não atingiu a assíntota (Figura 2), mantendo-se crescente ao longo de todo o período de amostragem, sinalizando que ainda existem espécies a serem amostradas no interior do Parque.

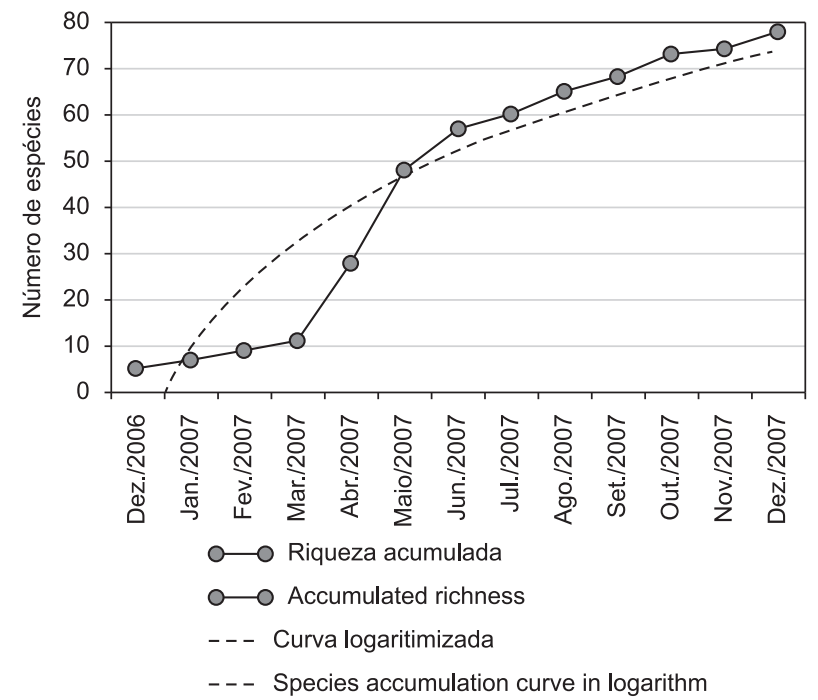

Figura 2. Curva de acúmulo de espécies de borboletas registradas no Parque Municipal Américo Renê Giannetti entre dezembro de 2006 e dezembro de 2007.

Figure 2. Species accumulation curve of butterflies recorded at the the Américo Renê Giannetti City Park between December 2006 and December 2007.
A partir das observações eventuais de visitação floral pelas borboletas adultas, constatou-se que a planta mais atrativa entre as observadas foi Lantana camara L. (Verbenaceae) (Tabela 2), observação também realizada por Lemes et al. (2008) ao estudarem visitantes florais no Jardim Botânico da Universidade Federal de Santa Maria, Rio Grande do Sul. Mesmo plantas incomuns em jardins ornamentais, como Asclepias curassavica L. (Apocynaceae) e Heliotropium indicum L. (Boraginaceae), são eficientes na atração destes insetos. Esta última, de acordo com Freitas et al. (2003), atraem várias borboletas da tribo Ithomiini e ambas atraem borboletas da subfamília Danainae. Devido a esta estreita interação entre borboletas e vegetação, a presença de plantas hospedeiras favorece o aumento da riqueza e abundância de espécies raras de borboletas, podendo ampliar a ocorrência destas espécies e contribuir com a conectividade entre áreas fragmentadas (Jonsen \& Lenore 1997).

Apesar de ainda haver poucas pesquisas com borboletas em fragmentos de mata urbanos, os estudos realizados em ambientes mergulhados em uma matriz aparentemente inóspita revelaram dados interessantes. Em Porto Alegre, Ruszczyk (1986a) registrou 43 espécies de borboletas ao longo das principais avenidas da cidade. Bonfantti et al. (2011) registraram 166 espécies em dois parque urbanos na cidade de Curitiba. Já na capital mineira, Silva et al. (2007) realizaram um estudo em uma mata urbana registrando 91 espécies de borboletas, o que corresponde a $18,7 \%$ da lepidopterofauna conhecida para Belo Horizonte (Brown \& Freitas 2000a). O presente trabalho, com 78 espécies de borboletas coletadas, representa $16 \%$ da fauna de Belo Horizonte, sendo que 34 espécies foram comuns entre o PMARG e a mata amostrada por Silva et al. (2007). Todas estas áreas demonstraram possuir condições microclimáticas e nichos ecológicos diversos que possibilitam a conservação e manutenção da riqueza de borboletas em grandes centros urbanos.

Estudos que correlacionam fragmentos de mata urbanos e lepidópteros revelam outros fatores ecológicos, além dos já conhecidos recursos alimentares e perturbações antrópicas, que influenciam a permanência de borboletas nestes ambientes. Ruszczyk (1986b) investigou a distribuição de borboletas ao longo dos gradientes de urbanização bem como a influência do habitat urbano na abundância destes insetos. $\mathrm{O}$ autor concluiu que a distância do centro da cidade foi a variável que mais influenciou na abundância e diversidade das espécies. Se confrontarmos a riqueza amostrada por Silva et al. (2007) com os resultados do presente estudo, concluiremos que foram registradas 13 espécies a menos no PMARG. Este localizase no centro da cidade de Belo Horizonte, enquanto o estudo de Silva et al. (2007) foi desenvolvido em uma área afastada do centro da capital. Esta comparação corrobora Ruszczyk (1986b), que afirma que a diversidade de borboletas é inversamente proporcional ao crescimento urbano devido à redução de áreas naturais. Esta tendência deve-se ao desaparecimento de espécies não adaptadas à urbanização, possibilitando que espécies colonizadoras dominem o ambiente atingindo densidades populacionais muito altas (Brown \& Freitas 1999).

Além de receber influências antrópicas como a poda constante de árvores e a poluição urbana, o PMARG é utilizado como área de lazer pela população. Cerca de $42 \%$ das espécies de borboletas deste inventário estão associadas a ambientes antropizados, urbanos e/ou perturbados. Entre as espécies, destacam-se H.f.februa, A. a. roeselia, T. claudina, J. evarete, S. stelenes, E. albula, B. p. polydamas, P. orcus, H. e. phyllis, dentre outras borboletas capazes de permanecer em ambientes muito alterados (Brown 1992, Raimundo 2003). De acordo com Brown \& Freitas (2002), embora a maioria dos grupos de borboletas possa sobreviver em matrizes urbanas, muitos grupos são pouco representados ou ausentes em parques muito pequenos ou com vegetação homogênea. Afirmam ainda que famílias como 
Tabela 2. Espécies vegetais fornecedoras de néctar observadas no PMARG, entre dezembro de 2006 e dezembro de 2007 e espécies de borboletas que as visitaram. Table 2. Plant species observed in the supply of nectar in PMARG between December 2006 and December 2007 and species of butterflies that have visited.

\section{Lepidoptera}

(Família/espécie)

\section{Vegetais}

(Família/espécie)

\section{Hesperiidae}

Urbanus dorantes

Urbanus proteus proteus

\section{Lycaenidae}

Rekoa palegon

\section{Nymphalidae}

Agraulis vanillae maculosa

Anartia jatrophae

Danaus erippus

Dryas iulia alcionea

Eresia lansdorfi

Heliconius erato phyllis

Junonia evarete

Marpesia petreus

Mechanitis lysimnia lysimnia

Mechanitis polymnia casabranca

Methona themisto

Ortilia ithra

Siproeta stelenes

Tegosa claudina

\section{Papilionidae}

Battus polydamas polydamas

Heraclides anchisiades capys

Parides anchises nephalion

\section{Pieridae}

Ascia monuste orseis

Eurema albula albula

Eurema elathea

Phoebis neocypris

Phoebis philea philea

Phoebis sennae marcellina

\section{Riodinidae}

Eurybia pergaea

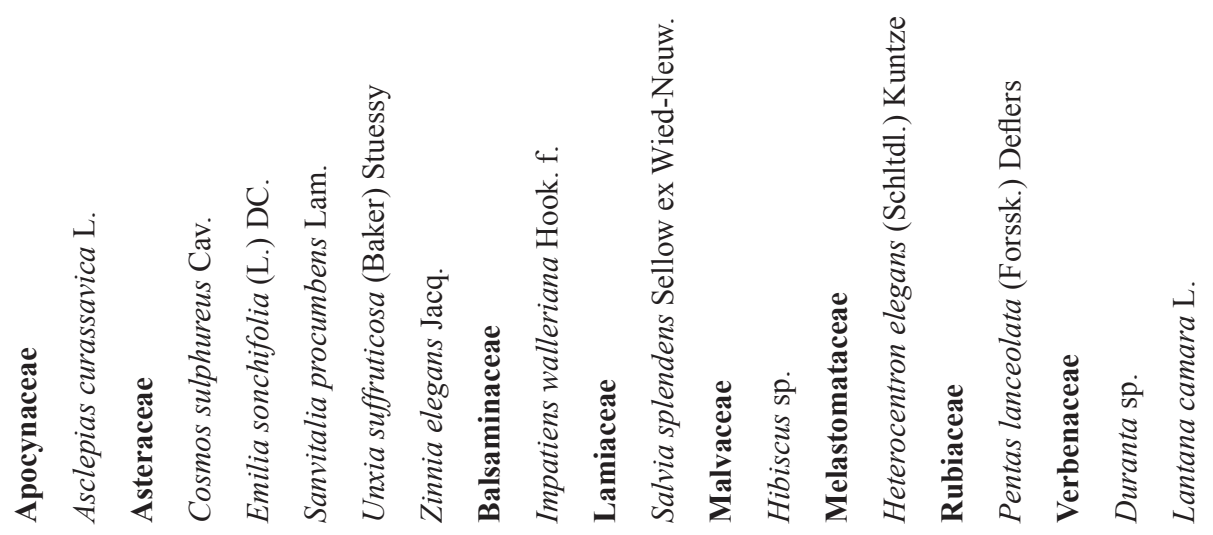

X

$\mathrm{X}$

$\mathrm{X}$

$\mathrm{X}$

$\mathrm{X}$

$\mathrm{X}$

$\mathrm{X}$

$\mathrm{X}$

$\mathrm{X}$

$\mathrm{X}$

$\mathrm{X}$

$\begin{array}{ll} & X \\ X & X \\ X & X \\ & X\end{array}$

X

$\mathrm{X}$

$\mathrm{X}$

$X \quad X$

$\mathrm{X}$

$\mathrm{X}$

$\mathrm{X}$

$\mathrm{X}$

X

$\mathrm{X}$

X

$\begin{array}{lll}X & & X \\ & & X \\ & X & X\end{array}$

$\mathrm{X}$

X

$\mathrm{X} \quad \mathrm{X}-\mathrm{X}$
Papilionidae, Nymphalidae (Morphinae, Charaxinae, Biblidinae, Satyrinae, Acreinae e Danainae), Pieridae (Pierinae), Hesperiidae e Lycaenidae, necessitam de espaço com vegetação heterogênea, água permanente e outros recursos especiais como formigas mutualísticas. No PMARG não se observou algumas destas subfamílias que necessitam de ambientes mais especializados para se desenvolverem como, por exemplo, Pyrrhopyginae (Hesperiidae). Grupos como estes são especialistas em recursos específicos que possuem fidelidade de microhabitat e respondem de maneira rápida à degradação de habitats (Brown 1991).

Uma das medidas prioritárias para a conservação de borboletas é a preservação das plantas utilizadas na alimentação das larvas e 
adultos, bem como a manutenção e/ou incorporação de plantas nos locais de ocorrência das borboletas. Fatores como conectividade, presença de corpos d'água permanentes, vegetação, flores, tamanho do fragmento e impactos antrópicos são os que mais influenciam a riqueza de borboletas em remanescentes urbanos e semi-urbanos (Brown \& Freitas 2002). Fortunato \& Ruszczyk (1997) afirmam que é de suma importância a preservação de fragmentos de vegetação nativa próximo às cidades para a manutenção da diversidade de borboletas em áreas urbanas. Algumas espécies de borboletas dispersam de seu habitat original em áreas florestadas maiores nos períodos de pico de crescimento populacional, parando em manchas de flores ou frutos e em áreas verdes, seguindo cursos de água ou ruas com árvores em linha verde (Brown \& Freitas 2002, Marini-Filho \& Martins 2010).

O monitoramento das espécies de borboletas se mostra uma ferramenta potencial para o fornecimento de informações relevantes para que medidas acertadas de conservação sejam tomadas antes que os efeitos da perturbação sejam irreversíveis. Preservar parques e áreas verdes urbanas, bem como manter plantas atrativas em seus jardins, como L. camara, proporcionam condições favoráveis para a permanência das comunidades de borboletas. Isto demonstra a importância da preservação do Parque Municipal Américo Renê Giannetti, dentro de uma matriz urbana. Do mesmo modo, conectar os fragmentos através do aumento de ruas arborizadas, controlar a poluição, ampliar os cursos d'água e manter o ambiente heterogêneo, se faz necessário para a conservação não apenas de borboletas, mas também de outros grupos animais.

\section{Agradecimentos}

Aos senhores Fernando Corrêa Campos Neto e Ivan Assunção Pimenta da Fundação Zoo-Botânica de Belo Horizonte. Também somos gratos a Onildo João Marini Filho e Danilo Bandini Ribeiro pela leitura crítica do manuscrito.

\section{Referências Bibliográficas}

BECCALONI, G.W. \& GASTON, K.J. 1995. Predicting species richness of Neotropical forest butterflies: Ithomiinae (Lepidoptera: Nymphalidae) as indicators. Biol. Conserv. 71(1):77-86. http://dx.doi.org/10.1016/00063207(94)00023-J

BONFANTTI, D., LEITE, L.A.R., CARLOS, M.M., CASAGRANDE, M.M., MIELKE, E.C. \& MIELKE, O.H.H. 2011. Riqueza de borboletas em dois parques urbanos de Curitiba, Paraná, Brasil. Biota Neotrop. 11(2): http://www.biotaneotropica.org.br/v11n2/pt/abstract?inventory+ bn00911022011 (último acesso em 12/07/2011).

BROWN, K.S. 1991. Conservation of neotropical environments: insects as indicators. In The conservation of insects and their habitats (N.M. Collins \& J.A. Thomas, eds.). Royal Entomological Society Symposium XV, London, Academic Press, p.349-404.

BROWN, K.S. 1992. Borboletas da Serra do Japi: diversidade, habitats, recursos alimentares e variação temporal. In História natural da Serra do Japi: ecologia e preservação de uma área florestal no sudeste do Brasil (L.P.C. Morellato, ed.). Unicamp, Fapesp, Campinas, p.142-187.

BROWN, K.S. 1996. Diversity of Brazilian Lepidoptera: history of study, methods for measurements, and use as indicator for genetic, specific and system richness. In Biodiversity in Brazil: a first approach (C.E.M. Bicudo \& N.A. Menezes, eds.). Instituto de Botânica, CNPq, São Paulo, p.221-253.

BROWN, K.S. \& FREITAS, A.V.L. 1999. Lepidoptera. In Biodiversidade do Estado de São Paulo, Brasil: síntese do conhecimento ao final do século XX, 5: invertebrados terrestres (C.R. Brandão \& E.M. Cancello, eds). FAPESP, São Paulo, p.226-243 http://www.biota.org.br/pdf/v5cap22.pdf (último acesso 12/07/2011).

BROWN, K.S. \& FREITAS, A.V.L. 2000a. Diversidade de Lepidoptera em Santa Teresa, Espírito Santo. Bol. Mus. Biol. Mello Leitão 11(12):71-118.
BROWN, K.S. \& FREITAS, A.V.L. 2000b. Atlantic Forest butterflies: indicators for landscape conservation. Biotropica 32(4b):150-172. http:// dx.doi.org/10.1111/j.1744-7429.2000.tb00631.x

BROWN, K.S. \& FREITAS, A.V.L. 2002. Butterfly communities of urban forest fragments in Campinas, São Paulo, Brazil: Structure, instability, environmental correlates, and conservation. J. Insect Conserv. 6:217-231. http://dx.doi.org/10.1023/A:1024462523826

CALLAGHAN, C.J. 1978. Studies on resting butterflies. II Notes on the population structure of Menander felsina (Riodinidae). J. Lepid. Soc. 32(1):37-48.

CANALS, G.R. 2003. Mariposas de Misiones. Literature of Latin America, Buenos Aires.

D'ABRERA, B. 1981. Butterfly of the Neotropical Region. Part I. Papilionidae \& Pieridae. Victoria, Hill House.

D'ABRERA, B. 1994. Butterfly of the Neotropical Region. Part VI. Riodinidae. Victoria, Hill House.

D'ABRERA, B. 1995. Butterfly of the Neotropical Region. Part VII. Lycaenidae. Victoria, Hill House.

DEVRIES, P.J. 1987. The butterflies of Costa Rica and their natural history. Princeton University Press, New Jersey.

DESSUY, M.B. \& MORAIS, A.B.B. 2007. Diversidade de borboletas (Lepidoptera, Papilionoidea e Hesperioidea) em fragmentos de Floresta Estacional Decidual em Santa Maria, Rio Grande do Sul, Brasil. Revta. Bras. Zool. 24(1):108-120. http://dx.doi.org/10.1590/S010181752007000100014

DIDHAM, R.K., GHAZOUL, J., STORK, N.E. \& DAVIS, A.J. 1996. Insects in fragmented forests: a functional approach. Trends Ecol. Evol. 11(6):255260. http://dx.doi.org/10.1016/0169-5347(96)20047-3

EMERY, E.O., BROWN, K.S. \& PINHEIRO, C.E.G. 2006. As borboletas (Lepidoptera, Papilionoidea) do Distrito Federal, Brasil. Rev. Bras. Entomol. 50(1):85-92. http://dx.doi.org/10.1590/S008556262006000100013

FRANCINI, R.B. 2010. História Natural das Borboletas do Vale do Rio Quilombo, Santos, SP. Edição do autor, Santos.

FORTUNATO, L. \& RUSZCZYK, A. 1997. Comunidades de Lepidópteros frugívoros em áreas verdes urbanas e extra-urbanas de Uberlândia, MG. Rev. Bras. Biol. 57:79-87.

FREITAS, A.V.L., FRANCINI, R.B. \& BROWN, K.S. 2003. Insetos como indicadores ambientais. In Métodos de estudo em Biologia da conservação e manejo da vida silvestre (L. Cullen, R. Rudran \& C. Valladares-Padua, orgs.). UFPR, Curitiba, p.125-151.

FREITAS, A.V.L. \& MARINI-FILHO, O.J. 2011. Plano de ação Nacional para conservação dos Lepidópteros ameaçados de extinção. ICMBio, Brasília, p.15-72. http://www.icmbio.gov.br/portal/images/stories/docsplano-de-acao/pan-lepidoptera/livro_lepidopteras_web2.pdf (último acesso em 18/12/2011).

HEPPNER, J.B. 1991. Faunal regions and the diversity of Lepidoptera. Trop. Lepid. 2(1):1-85.

INSTITUTO NACIONAL DE METEREOLOGIA - INMET. http:// www.inmet.gov.br/html/clima/mapas/?mapa=prec (último acesso em 12/07/2011) .

ISERHARD, C.A. \& ROMANOWSKI, H.P. 2004. Lista de espécies de borboletas (Lepidoptera, Papilionoidea e Hesperioidea) da região do vale do rio Maquiné, Rio Grande do Sul, Brasil. Rev. Bras. Zool. 21(3):649-662. http://dx.doi.org/10.1590/S0101-81752004000300027

ISERHARD, C.A., QUADROS, M.T., ROMANOWSKI, H.P. \& MENDONÇA JUNIOR, M.S. 2010. Borboletas (Lepidoptera: Papilionoidea e Hesperioidea) ocorrentes em diferentes ambientes na floresta Ombrófila Mista e nos Campos de Cima da Serra do Rio Grande do Sul, Brasil. Biota Neotrop. 10(1): http://www.biotaneotropica.org.br/v10n1/pt/abstract?inv entory+bn02910012010 (último acesso em 12/07/2011). 
JONSEN, I.D. \& LENORE, F. 1997. Response of generalist and specialist insect herbivores to landscape spatial structure. Landscape Ecol. 12:185-197. http://dx.doi.org/10.1023/A:1007961006232

KOH, L.P. \& SODHI, N.S. 2004. Importance of reserves, fragments, and parks for butterfly conservation in tropical urban landscape. Ecol. Appl. 14(6):1698-1708. http://dx.doi.org/10.1890/03-5269

LAMAS, G. 2004. Checklist: Part 4A. Hesperioidea-Papilionoidea. In Atlas of Neotropical Lepidoptera. (J.B. Heppner, ed.). Association for Tropical Lepidoptera/Scientific Publishers, Gainesville, p.1-439.

LEMES, R., RITTER, C.D. \& MORAIS, A.B.B. 2008. Borboletas (Lepidoptera: Hesperioidea e Papilionoidea) visitantes florais no Jardim botânico da Universidade Federal de Santa Maria, Santa Maria, RS, Brasil. Rev. Biotemas 21(4):91-98.

LEPPIK, E.E. 1957. Evolutionary relationship between entomophilous plants and anthophilous insects. Evolution 11(4):466-481. http://dx.doi. org $/ 10.2307 / 2406067$

LEWINSOHN, T.M., FREITAS, A.L.V. \& PRADO, P.I. 2005. Conservação de invertebrados terrestres e seus habitats no Brasil. Megadiversidade 1(1):6269.

LORENZI, H. \& SOUZA, H.M. 1995. Plantas Ornamentais no Brasil: arbustivas, herbáceas e trepadeiras. Editora Plantarum, São Paulo, 720p.

MARCHIORI, M.O. \& ROMANOWSKI, H.P. 2006. Borboletas (Lepidoptera, Papilionoidea e Hesperioidea) do Parque Estadual do Espinilho e entorno, Rio Grande do Sul, Brasil. Rev. Bras. Zool. 23(4):1029-1037. http:// dx.doi.org/10.1590/S0101-81752006000400007

MARINI-FILHO, O.J. \& MARTINS, R.P. 2010. Nymphalid butterfly dispersal among forest fragments at Serra da Canastra National Park, Brazil. J. Insect Conserv. 14(4):401-411. http://dx.doi.org/10.1007/ s10841-010-9271-9

MURPHY, D.D. 1997. Desafios à diversidade biológicas em áreas urbanas. In Biodiversidade (E.O. Wilson. ed.). Nova Fronteira, Rio de Janeiro, p.89-97.

NEW, T.R., PYLE, R.M., THOMAS, J.A., THOMAS, C.D. \& HAMMOND, P.C. 1995. Butterfly conservation and management. Ann. Rev. Ecol. Evol. Syst. 40:56-83.

OTERO, L.S. 1986. Borboletas - O Livro do Naturalista. FAE, Rio de Janeiro.

PAZ, A.L.G., ROMANOWSKI, H.P. \& MORAIS, A.B.B. 2008. Nymphalidae, Papilionidae e Pieridae (Lepidoptera: Papilionoidea) da Serra do Sudeste do Rio Grande do Sul, Brasil. Biota Neotrop. 8(1): http://www. biotaneotropica.org.br/v8n1/pt/abstract?inventory+bn01608012008 (último acesso em 18/12/2011).

PRICE, P.W. 1984. Insect Ecology. John Wiley \& Sons, New York.

PRIMACK, R.B. \& RODRIGUES, E. 2001. Biologia da Conservação. Planta, Londrina.

POLLARD, E. 1977. A method for assessing changes in the abundance of butterflies. Biol. Conserv. 12:115-134. http://dx.doi.org/10.1016/00063207(77)90065-9
RAIMUNDO, R.L.G., FREITAS, A.V.L., COSTA, R.N.S., OLIVEIRA, J.B.F., LIMA, A.F., MELO, A.B. \& BROWN, K.S. 2003. Manual de monitoramento ambiental usando borboletas e libélulas - Reserva extrativista do Alto Juruá. Série Pesquisa e Monitoramento Participativo em áreas de conservação gerenciadas por populações tradicionais. CERES/Laboratório de Antropologia e Ambiente, Campinas, v.1.

ROSA, P.L.P., CHIVA, E.Q. \& ISERHARD, C.A. 2011. Borboletas (Lepidoptera: Papilionoidea e Hesperioidea) do sudoeste do Pampa brasileiro, Uruguaiana, Rio Grande do Sul, Brasil. Biota Neotrop. 11(1): $\mathrm{http} / / /$ www.biotaneotropica.org.br/v11n1/en/abstract?inventory+ bn00411012011 (último acesso em 18/12/2011).

RUSZCZYK, A. 1986a. Ecologia urbana de borboletas, I. O gradiente de urbanização e a fauna de Porto Alegre RS. Rev. Bras. Biol. 46:675-688.

RUSZCZYK, A. 1986b. Distribution and abundance of butterflies in the urbanization zones of Porto Alegre, Brazil. J. Res. Lepid. 25:157-178.

RUSZCZYK, A. \& CARVALHO, M.C. 1993. Malfunction of ecdysis and female biased mortality in urban Brassolis sophorae (Nymphalidae: Brassolinae). J. Lepid. Soc. 47(2):134-139.

SACKIS, G.D. \& MORAIS, A.B.B. 2008. Borboletas (Lepidoptera: Hesperioidea e Papilionoidea) do campus da Universidade Federal de Santa Maria, Santa Maria, Rio Grande do Sul. Biota Neotrop. 8(1): http:// www.biotaneotropica.org.br/v8n1/pt/abstract?inventory+bn01908012008 (último acesso em 12/07/2011).

SANTOS, E.C., MIELKE, O.H.H. \& CASAGRANDE, M.M. 2008.Inventários de borboletas no Brasil: estado da arte e modelo de áreas prioritárias para pesquisa com vistas à conservação. Nat. Conserv. 6(2):68-90.

SILVA, A.R.M., LANDA, G.G. \& VITALINO, R.F. 2007. Borboletas (Lepidoptera) de um fragmento urbano em Minas Gerais, Brasil. Lundiana 8(2):137-142.

SOUZA, V.C. \& LORENZI, H. 2005. Botânica sistemática: guia ilustrado para identificação das famílias de Angiospermas da flora brasileira, baseado em APG II. Instituto Plantarum, Nova Odessa, Brasil.

STEBBINS, G.L. 1970. Adaptative radiation of reproductive characteristics in angiosperms: pollination mechanisms. Ann. Rev. Ecol. Sys. 1:307-325. http://dx.doi.org/10.1146/annurev.es.01.110170.001515

STEVENS, P.F. 2001. Angiosperm Phylogeny Website. versão 12 de Julho de 2012. http://www.mobot.org/MOBOT/research/APweb/ (último acesso em 27/09/2012).

TYLER, H.A., BROWN, K.S. \& WILSON, K.H. 1994. Swallowtail Butterflies of the Americas: A study in Biological Dynamics, Ecological Diversity, Biosystematics and Conservation. Scientific Publishers, Gainesville.

WILLMOTT, K.R. 2003. The genus Adelpha: Its systematic, biology and biogeography (Lepidoptera Nymphalidae: Limenitidini). Scientific Publishers, Gainesville.

WAHLBERG, N., LENEVEU, J., KODANDARAMAIAH, U., PEÑA, C., NYLIN, S., FREITAS, A.V.L. \& BROWER, A.V.Z. 2009. Nymphalidae butterflies diversify following near demise at the cretaceous/ tertiary boundary. Proc. R. Soc. B. 276:4295-4302. PMid:19793750 PMCid:2817107. http://dx.doi.org/10.1098/rspb.2009.1303

WILSON, E.O. 2003. Biodiversity. National Academy Press, Washington. 